\title{
Single Centre Experience with Minimally Invasive Aortic Valve Replacement versus Conventional Full Sternotomy Approach - A Propensity Match Analysis
}

\author{
Karim Morcos ${ }^{1 *}$, Cathy Johnman², Cristiano Spadaccio ${ }^{1}$, Sadia Aftab ${ }^{1}$, Fraser Sutherland ${ }^{1}$ \\ From World Society of Cardiothoracic Surgeons 25th Anniversary Congress, Edinburgh \\ Edinburgh, UK. 19-22 September 2015
}

\section{Background/Introduction}

Minimally invasive approach to aortic valve replacement (AVR) is increasingly accepted as a valid alternative to conventional full sternotomy (FS-AVR), as reduces operative trauma with the final aim to improve the postoperative outcomes.

\section{Aims/Objectives}

The aim of our study is to compare short term clinical outcomes after minimally invasive AVR (mini-AVR) with outcomes following FS-AVR in the same institution.

\section{Method}

Between December 2010 and March 2012627 patients underwent isolated AVR were retrospectively included in two groups: 599 patients underwent FS-AVR sternotomy (Group A), while 28 underwent minimally invasive procedure (Group B). Mini-AVR was performed through a $6 \mathrm{~cm}$ upper midline incision with reverse 'J' manubriotomy carried into the right third intercostal space. Venous drainage for cardiopulmonary bypass was achieved alternatively percutaneously or with a flat two stage venous cannula with vacuum assist. Primary endpoint was periprocedural mortality; secondary endpoints were overall postoperative complications, major adverse cardiacrelated complication, use of blood products and need for transfusions, bypass time and cross-clamp time, ventilation time and length of stay in hospital. Propensity score match analysis was performed to avoid selection biases and equalize confounding preoperative variables.

\section{Results}

After propensity score match, no statistical significant difference was found in peri-procedural mortality rate ( $p>0.05$ ), mean bypass and cross clamp times. Minimally invasive AVR was associated with a significant reduced need for transfusion $(\mathrm{p}=0.003)$, as well as postoperative cardiac and non-cardiac complications. A trend towards lower mean ventilation times, ICU stay and hospital stay in the mini-AVR group was also detected, but failed to reach statistical significance.

\section{Discussion/Conclusion}

Initial results with minimally invasive AVR are associated with significantly reduced blood loss, reduced blood transfusion and a trend towards less ventilation time, ICU stay and hospital stay. Postoperative cosmetic results were much better in the minimally invasive group.

\section{Authors' details \\ ${ }^{1}$ Department of Cardiothoracic Surgery, Golden Jubilee National Hospital, Glasgow, Scotland, UK. ${ }^{2}$ Institute of Health and Wellbeing, College of Medical, Veterinary and Life Sciences, Public Health University of Glasgow, Glasgow, Scotland, UK.}

Published: 16 December 2015

doi:10.1186/1749-8090-10-S1-A109

Cite this article as: Morcos et al: Single Centre Experience with Minimally Invasive Aortic Valve Replacement versus Conventional Full Sternotomy Approach - A Propensity Match Analysis. Journal of Cardiothoracic Surgery 2015 10(Suppl 1):A109. 